\title{
TITLE:
}

\section{Geometry of one-dimensional wave propagation}

$\operatorname{AUTHOR}(S)$ :

Kitano, M.

\section{CITATION:}

Kitano, M.. Geometry of one-dimensional wave propagation. Physical Review A 1995, 51(6): 4427-4432

ISSUE DATE:

1995-06

URL:

http://hdl.handle.net/2433/152444

RIGHT:

(C) 1995 The American Physical Society 


\title{
Geometry of one-dimensional wave propagation
}

\author{
M. Kitano \\ Department of Electronics, Kyoto University, Kyoto 606-01, Japan
}

(Received 6 October 1994)

\begin{abstract}
We investigate the geometrical features of one-dimensional wave propagation, whose dynamics is described by the $(2+1)$-dimensional Lorentz group. We find many interesting geometrical ingredients such as spinorlike behavior of wave amplitudes, gauge transformations, Bloch-type equations, and Lorentz-group Berry phases. We also propose an optical experiment to verify these effects.
\end{abstract}

PACS number(s): 03.65.Bz, 03.40.Kf, 02.40.-k

\section{INTRODUCTION}

The Berry phases [1] produced by rotations have been most extensively studied for various physical systems. Another interesting class consists of Berry phases produced by Lorentz transformations. Chiao and Jordan [2] showed that Lorentz-group Berry phases could be observed as a change of phase of the electromagnetic field in squeezed states. Successive squeezing operations for either light or microwaves by degenerate parametric amplifiers induce a phase shift that can be interpreted geometrically. These kinds of experiments have drawn considerable interest because they can be viewed as the quantum optical realization of the Lorentz kinematics [3,4].

Another optical manifestation of the Lorentz-group Berry's phase was proposed and experimentally verified [5]. It is shown that when one cycles light through a sequence of partially polarized states, the light acquires a geometrical phase.

It is well known that the wave propagation in one dimension (1D) can be described by the Lorentz-group dynamics, therefore, one can expect Lorentz-group Berry phases in wave systems. In this paper, we present Berry phases and related geometrical features seen in the 1D wave propagation in inhomogeneous media.

In Sec. II, from the 1D wave equation with quasiperiodic potential we derive a Schrödinger-like equation, which explicitly exhibits the Lorentz dynamics via the generators of $\operatorname{SL}(2, \mathbb{R})$. The dynamical variables of the equation are the two quadrature wave amplitudes, which form a two-column state vector.

In Sec. III, a $\mathrm{SL}(2, \mathbb{R})$-Bloch equation is derived from the Schrödinger-like equation. The equation is very similar to the conventional SU(2)-Bloch equation for the spin$1 / 2$ except that the $\mathrm{SL}(2, \mathbb{R})$-Bloch vector moves on a conical surface not on a sphere. The similarity could be very useful for the geometrical understanding of Lorentz dynamics, especially because the $\mathrm{SU}(2)$-Bloch model has been successfully employed in various fields such as nuclear magnetic resonances, nonlinear optics, polarization optics, interferometry, and atomic physics, etc.

In Sec. IV, we discuss two-valuedness of the state vectors. A Bloch vector represents a pair of state vectors with opposite signs. When a Bloch vector circles around the apex of a cone, the corresponding state vec- tor changes its sign. It is shown that the two-valuedness comes from a symmetry of the wave equation with respect to the translation of half wavelength.

Extending the above geometrical situation, we introduce in Sec. V a local gauge transformation associated with the deformation of space coordinate.

So far, we have assumed that the state vector is real because the potential function in the wave equation is real. It turns out, however, the extension to complex wave functions is very convenient, because a complex solution could represent two linearly independent real solutions at once. In Sec. VI, we derive a Bloch equation for the case of complex amplitudes. In this case, a Bloch vector corresponds to a set of state vectors that differ only in phase factors. The Bloch vector moves on the surface of hyperboloid. This is the arena where the Berry phase plays a role. In Sec. VII, we define the Berry phase for a cyclic evolution of the system.

Finally, we propose a feasible optical experiment where we can observe the Berry phase for $1 \mathrm{D}$ wave propagation.

\section{1D WAVE EQUATION AND SL(2,R)}

Let us start with a one-dimensional wave equation (the Helmholtz equation) for a monochromatic wave $\Psi(x) \mathrm{e}^{-i \omega t}$

$$
\left\{\frac{d^{2}}{d x^{2}}+k^{2}[1+\epsilon(x)]\right\} \Psi(x)=0,
$$

where $k=k(\omega)$ is the wave number in free $(\epsilon=0)$ space.

We assume the spatial variation of the refractive index or the potential $\epsilon(x)$ has the following form:

$$
\epsilon(x)=\epsilon_{0}(x)+2 \epsilon_{c}(x) \cos 2 k x+2 \epsilon_{s}(x) \sin 2 k x .
$$

The spatial modulation at wave number $2 k$ strongly modifies the propagation of the wave with wave number $k$ owing to the Bragg effect. The dc component $\epsilon_{0}$ also has substantial influences through the Bragg resonance condition. $k$ as

We represent the wave $\Psi(x)$ with mean wave number

$$
\Psi(x)=A(x) \cos k x+B(x) \sin k x,
$$


where $A(x)$ and $B(x)$ are slowly varying envelope functions. These envelopes are normally considered to be real functions, since $\epsilon(x)$ in Eq. (1) is real. However, the extension to complex functions is sometimes very useful as will be discussed in Sec. VI.

Substitution of Eqs. (2) and (3) into Eq. (1) gives

$$
\frac{d}{d x}\left[\begin{array}{l}
A \\
B
\end{array}\right]=\frac{k}{2}\left[\begin{array}{cc}
\epsilon_{s} & -\epsilon_{c}+\epsilon_{0} \\
-\epsilon_{c}-\epsilon_{0} & -\epsilon_{s}
\end{array}\right]\left[\begin{array}{l}
A \\
B
\end{array}\right]
$$

which can be rewritten in a Schrödinger-like form,

$$
i k^{-1} \frac{d}{d x}|\psi\rangle=-\boldsymbol{\epsilon}(x) \cdot \boldsymbol{K}|\psi\rangle
$$

with the column vector $|\psi\rangle=(A, B)^{T}$. Here we introduced $\epsilon(x)=\left(-\epsilon_{s}, \epsilon_{c}, \epsilon_{0}\right)=\left(\epsilon_{1}, \epsilon_{2}, \epsilon_{3}\right)$, and $\boldsymbol{K}=$ $\left(K_{1}, K_{2}, J_{3}\right)$, with

$$
K_{1}=i \sigma_{3} / 2, K_{2}=i \sigma_{1} / 2, J_{3}=\sigma_{2} / 2,
$$

where $\sigma_{i}(i=1,2,3)$ are the Pauli's spin matrices.

We have assumed that $A(x)$ and $B(x)$ do not change appreciably over distances of the order of $k^{-1}$ (slowly-varying-envelope approximation) and neglected the terms with wavelength $\pm 3 k$ (secular-term approximation).

The matrices (6) are the generators of $\operatorname{SL}(2, \mathbb{R})$, the two-dimensional real unimodular group, and satisfy the commutation relations

$$
\left[K_{1}, K_{2}\right]=-i J_{3}, \quad\left[K_{2}, J_{3}\right]=i K_{1}, \quad\left[J_{3}, K_{1}\right]=i K_{2} .
$$

The $(A, B)$ plane is squeezed in one direction and stretched in the orthogonal direction by the generators $K_{1}$ or $K_{2}$, and rotated by $J_{3}$.

The group $\mathrm{SL}(2, \mathbb{R})$ has the close connection (locally isomorphic) to the $(2+1)$-dimensional Lorentz group $\mathrm{SO}(2,1)$ as does the group $\mathrm{SU}(1,1)$. Instead of Eq. (3), if we use a representation

$$
\Psi(x)=\mathcal{A}(x) e^{-i k x}+\mathcal{B}(x) e^{i k x},
$$

then we have the $\mathrm{SU}(1,1)$-Schrödinger equation with $K_{1}^{\prime}=i \sigma_{1} / 2, K_{2}^{\prime}=-i \sigma_{2} / 2, J_{3}^{\prime}=\sigma_{3} / 2$, which satisfy the same commutation relations as Eq. (7). In the following discussion, we mostly use $\operatorname{SL}(2, \mathbb{R})$ rather than $\mathrm{SU}(1,1)$.

\section{SL $(2, \mathbb{R})$-BLOCH EQUATION - REAL AMPLITUDE CASE}

Equation (5) has a form similar to the Schrödinger equation for the spin-1/2 $(\boldsymbol{J})$ in time varying magnetic fields $\boldsymbol{B}(t) ; i \hbar(d / d t)|\psi\rangle=-\boldsymbol{B}(t) \cdot \boldsymbol{J}|\psi\rangle$. The underlying group for the spin-1/2 dynamics is $\mathrm{SU}(2)$, which is the covering group of the three-dimensional rotation group, $\mathrm{SO}(3)$. The Bloch equation derived from the spin-1/2 Schrödinger equation apparently reflects the structure of those groups; the Bloch vector moves on a sphere, and the Berry phase for spin $1 / 2$ can be related to the surface area of the sphere.
In order to see the geometrical structure of $\operatorname{SL}(2, \mathbb{R}) \mathrm{dy}$ namics, let us derive the corresponding Bloch equation. (A more detailed derivation will be given in Sec. VI. See also Ref. [6].)

First we define the $2 \times 2$ density matrix $\rho$ as

$$
\rho=2 i|\psi\rangle\langle\psi| J_{3}=i s \cdot \boldsymbol{K}
$$

where $\langle\psi|$ represents the row vector $(A, B)$, and $s=$ $\left(s_{1}, s_{2}, s_{3}\right)$ the Bloch vector. We note,

$$
\mathrm{s}=\left(2 A B,-A^{2}+B^{2}, A^{2}+B^{2}\right) .
$$

The equation of motion for $\rho$ can be obtained from Eq. (5) as

$$
i k^{-1} \frac{d \rho}{d x}=[-\boldsymbol{\epsilon} \cdot \boldsymbol{K}, \rho] .
$$

Using the commutation relations (7), we have the equation of motion for $s$,

$$
k^{-1} \frac{d s}{d t}=\epsilon \tilde{\times} \boldsymbol{s}
$$

where the $\mathrm{SU}(1,1)$ vector product defined as

$$
\boldsymbol{a} \tilde{\times} \boldsymbol{b}=\left(a_{2} b_{3}-a_{3} b_{2}, a_{3} b_{1}-a_{1} b_{3},-a_{1} b_{2}+a_{2} b_{1}\right)
$$

for two vectors, $\boldsymbol{a}=\left(a_{1}, a_{2}, a_{3}\right)$ and $\boldsymbol{b}=\left(b_{1}, b_{2}, b_{3}\right)$. We also define the $\mathrm{SU}(1,1)$ scalar product,

$$
\boldsymbol{a} \sim \boldsymbol{b}=a_{1} b_{1}+a_{2} b_{2}-a_{3} b_{3} .
$$

Then from Eq. (10), we have

$$
\boldsymbol{s} \sim \boldsymbol{s}=0, \quad s_{3} \geq 0
$$

which means the Bloch vector $s$ moves on the surface of the (upper) cone depicted in Fig. 1.

When $\epsilon(x)$ is constant, trajectories for $s$ are very simple; the vector $s$ lies in a plane that is orthogonal to the vector $\tilde{\boldsymbol{\epsilon}}=\left(\epsilon_{1}, \epsilon_{2},-\epsilon_{3}\right)$, because

$$
\frac{d \boldsymbol{s}}{d x} \sim \boldsymbol{\epsilon}=\frac{d \boldsymbol{s}}{d x} \cdot \tilde{\boldsymbol{\epsilon}}=0 .
$$

A trajectory, which is the intersection of a plane and the cone could be an ellipse, a parabola, or a hyperbola according as $\epsilon_{3}^{2}$ is greater than, equal to, or less than $\epsilon_{1}^{2}+\epsilon_{2}^{2}$. The elliptical, bound trajectories correspond to the propagating waves in the conduction bands and the hyperbolic, unbound trajectories correspond to the

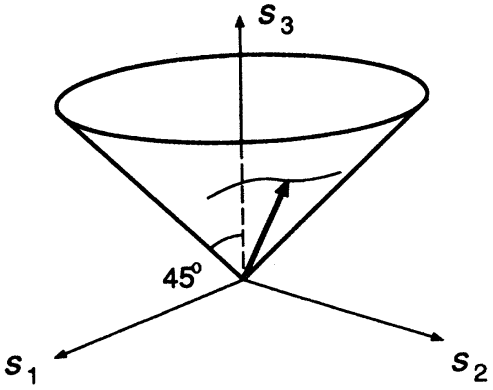

FIG. 1. Bloch vector $s$ (for real amplitudes) on the conic surface: $s_{1}^{2}+s_{2}^{2}-s_{3}^{2}=0$. 
evanescent waves in the forbidden bands.

Owing to the geometrical simplicity and the similarity to the conventional Bloch equation, the $\mathrm{SL}(2, \mathbb{R})$-Bloch equation could be very useful.

\section{TWO-VALUEDNESS}

In this section, we study a subtler geometrical feature of $\mathrm{SL}(2, \mathbb{R})$-Bloch equation. We note the correspondence between $(A, B)$ and $s$ given by Eq. (10) is not one to one but two to one; a single vector $s$ represents two state vectors with opposite signs: $(A, B)$ and $(-A,-B)$.

At first sight, these two state vectors may seem to represent two distinct physical situations. Within the framework of the present approximation (slowly varying envelopes), however, the two situations are virtually identical. Figure 2 represents the local pictures of waves with opposite polarities (real lines). A potential with period $\sim \pi k^{-1}$ is also shown (dashed lines). We note the dispositions of the waves relative to the potential are the same and, therefore, the waves would evolve identically.

This degeneracy comes from the fact that the potential (2) is invariant under the translation $x \rightarrow x+n \pi k^{-1}(n$ : integer), as far as the variation of $\epsilon(x)$ over the distance $n \pi k^{-1}$ can be neglected;

$$
\epsilon\left(x+n \pi k^{-1}\right) \sim \epsilon(x) .
$$

On the other hand, from Eq. (3), we see that the translation induces $(A, B) \rightarrow(-1)^{n}(A, B)$.

In order to see the geometrical structure of the twovalued representation, we can make a cone from the $(A, B)$ plane as shown in Fig. 3. The $(A, B)$ plane is cut along a line from the origin ( $+A$ axis in this example) and is wrapped to make a twofold cone with apex angle of 60 degrees. When the vector $s$ on the cone encircles a closed curve around the apex, we see that the corresponding $(A, B)$ gains a factor $(-1)^{n}$, where $n$ is the winding number of the curve.

Even though, two vectors $(A, B)$ and $(-A,-B)$ represent (almost) the same physical situation, we can conveniently detect the minus sign by interferometric methods, as that for spin $1 / 2$ rotated by $2 \pi[7]$. (The two cases have good analogy, but it is only for the latter case that interference experiments are absolutely required and even in principle there is no other way to detect the minus sign.)

Figure 4 shows an example of interferometry. We have

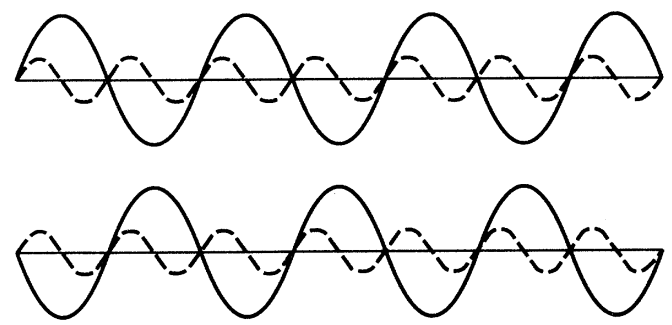

FIG. 2. Waves with opposite polarities (real lines) have the same phase relationship with the half-periodic potential (dashed line).
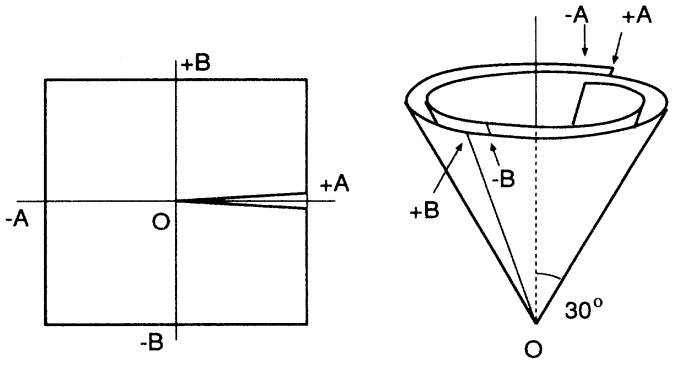

FIG. 3. In order to identify $(A, B)$ and $(-A,-B)$, which represent physically the same state, we make a twofold cone from the $(A, B)$ plane by making a cut from the origin and wrapping.

two trajectories both of which start from $(A, B)=(1,0)$ at $x=0$. One evolves with a constant $\epsilon$ and the other with $-\epsilon$. After some evolution, they reach conjugate points in the $(A, B)$ plane. Looking at the corresponding wave forms [Fig. 4(b)], we notice that the phase shifts accumulate to make signs opposite.

This situation reminds us of the sign change of a spinor rotated by $2 \pi$, the Aharonov-Bohm effect with an infinite solenoid, the sign change around the degeneracy of the eigenstates of real-Hamiltonian systems $[8,9]$, and the (relative) configuration space of two identical particles in two-dimensional space [10]. In terms of the second analogy, the topological magnetic flux of $\pi \hbar c / e$ is required to account for the sign change or the phase shift of $\pi$. The amount of flux is consistent with the (singular) curvature at the cone apex or the apex angle.

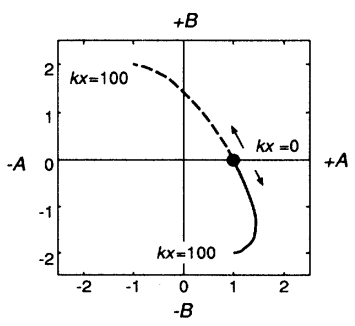

(a)

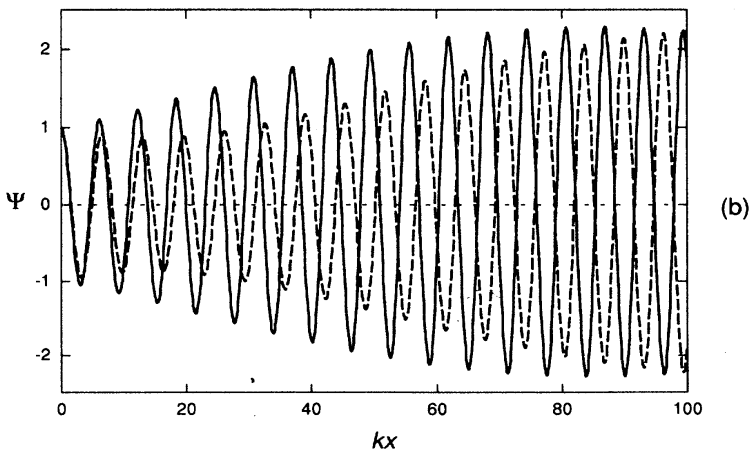

FIG. 4. Interferometric detection of two-valuedness of wave amplitudes. (a) Two trajectories starting off from $(A, B)=(1,0)$ at $x=0$ evolved by $\epsilon=\pi(-2,1,3) / 400$ (real line), and by $-\epsilon$ (dashed line), respectively, and reach conjugate points at $x=100 k^{-1}$. (b) Corresponding wave forms. 
Mathematically speaking, $\mathrm{SL}(2, \mathbb{R})$ is a two-valued representation of the $(2+1)$-dimensional Lorentz group, $\mathrm{SO}(2,1)$, as $\mathrm{SU}(2)$ is for the three-dimensional rotation group, $\mathrm{SO}(3)$. It is very interesting that the two-valued representation which is believed peculiar to the quantum regime manifests itself in the classical context.

It should be noted that the cone introduced here and that for the Bloch vector discussed in the previous section have different apex angles and different parametrization:

$$
\bar{s}=\left(s_{1}, s_{2}, \sqrt{3} s_{3}\right) / 2 \sqrt{s_{3}},
$$

where $\overline{\boldsymbol{s}}$ is a vector in Fig. 3.

\section{GAUGE TRANSFORMATION}

In order to generalize the discussion in the previous section, we introduce a (local) gauge transformation. We conveniently use the $\mathrm{SU}(1,1)$ amplitudes [see Eq. (8)] $\mathcal{A}$ and $\mathcal{E}$ :

$$
\mathcal{A}=(A+\mathrm{i} B) / 2, \quad \mathcal{E}=\epsilon_{c}+\mathrm{i} \epsilon_{s} .
$$

With this notation, the Schrödinger equation (5) can be represented in a scalar form as

$$
2 i k^{-1} \frac{d}{d x} \mathcal{A}=\mathcal{E} \mathcal{A}^{*}+\epsilon_{0} \mathcal{A}
$$

where $\mathcal{A}^{*}$ stands for the complex conjugate of $\mathcal{A}$.

Here we consider a space coordinate $x^{\prime}$ which is slightly deviated from $x$ as

$$
x^{\prime}=x-\xi(x)
$$

We assume the deviation $\xi$ is small enough to assure $\mathcal{A}(x-\xi) \sim \mathcal{A}(x), \mathcal{E}(x-\xi) \sim \mathcal{E}(x)$, and $\xi(x-\xi) \sim \xi(x)$ for any $x$. In the $x^{\prime}$ coordinate, $\mathcal{A}^{\prime}$ and $\mathcal{E}^{\prime}$ are defined as

$$
\mathcal{A}^{\prime}=\mathcal{A} e^{-i k \xi}, \quad \mathcal{E}^{\prime}=\mathcal{E} e^{-2 i k \xi},
$$

respectively, and the equation of motion transforms as

$$
2 i k^{-1} \frac{d}{d x^{\prime}} \mathcal{A}^{\prime}=\mathcal{E}^{\prime} \mathcal{A}^{\prime *}+\left[\epsilon_{0}+2 \frac{d \xi}{d x^{\prime}}\right] \mathcal{A}^{\prime}
$$

Here we have an extra term $2 d \xi / d x^{\prime}$ which accounts for the gauge transformation. This term can be interpreted as a gauge field.

In Eqs. (2) and (3), in order to define $\epsilon, A$, and $B$, we needed a long yardstick accurately graduated in $2 \pi k^{-1}$. However, with the help of the above equations, we can compare the wave amplitudes for two observers whose rulers are not necessarily accurate in a long span.

We note that the discussion in the previous section can be reproduced by considering the case of constant deviation $\xi(x)=n \pi k^{-1}$, which yields $\mathcal{A}^{\prime}=-\mathcal{A}, \mathcal{E}^{\prime}=\mathcal{E}$, and $d \xi / d x^{\prime}=0$. We consider two coordinate systems $x$ and $x^{\prime}$ shifted by $n \pi k^{-1}$, and a third coordinate system $x^{\prime \prime}=x+\xi(x)$, with $\xi(x)=0$ for $x<x_{1}$ and $\xi(x)=n \pi k^{-1}$ for $x>x_{2}$; in the interval of $x_{1}<x<x_{2}, x^{\prime \prime}$ smoothly connects $x$ and $x^{\prime}$. The integration of the gauge field

$$
l=\int_{x_{1}}^{x_{2}} 2 \frac{d \xi(x)}{d x} d x=2 n \pi k^{-1}
$$

which is independent of the local behavior of $\xi$, amount to the sign factor $\exp (-i n k l / 2)=(-1)^{n}$ between $(A, B)$ and $\left(A^{\prime}, B^{\prime}\right)$.

\section{SL(2, R) BLOCH EQUATION - COMPLEX AMPLITUDE CASE}

From now on let us consider $|\psi\rangle=(\alpha, \beta)^{T}$ as complex amplitudes. Before proceeding, physical meaning of complex amplitudes should be clarified. At first sight of Eq. (1), which has real coefficients, no complex amplitudes seems required. In fact, if we decompose $|\psi\rangle$ into real and imaginary parts,

$$
|\psi\rangle=\left|\psi^{\prime}\right\rangle+i\left|\psi^{\prime \prime}\right\rangle
$$

then each of $\left|\psi^{\prime}\right\rangle$ and $\left|\psi^{\prime \prime}\right\rangle$ is a real solution to Eq. (5). Therefore, a complex solution could represent two independent real solutions. The independence is assured by $\alpha^{\prime} \beta^{\prime \prime}-\alpha^{\prime \prime} \beta^{\prime} \neq 0$, where $\left|\psi^{\prime}\right\rangle=\left(\alpha^{\prime}, \beta^{\prime}\right)^{T}$ and $\left|\psi^{\prime}\right\rangle=\left(\alpha^{\prime \prime}, \beta^{\prime \prime}\right)^{T}$. A complex solution is convenient in the sense that any real solutions can be represented as $\operatorname{Re}(z|\psi\rangle)$ with a complex number $z$.

Starting from the $\mathrm{SL}(2, \mathbb{R})$-Schrödinger equation (5) with complex $|\psi\rangle$ :

$$
\begin{array}{r}
i k^{-1} \frac{d}{d x}|\psi\rangle=N(x)|\psi\rangle, \\
N(x)=\epsilon_{1} K_{1}+\epsilon_{2} K_{2}+\epsilon_{3} J_{3},
\end{array}
$$

let us derive the corresponding Bloch-like equation. The adjoint equation [11] is

$$
\begin{array}{r}
i k^{-1} \frac{d}{d x}|\varphi\rangle=N^{\dagger}(x)|\varphi\rangle, \\
N^{\dagger}(x)=-\epsilon_{1} K_{1}-\epsilon_{2} K_{2}+\epsilon_{3} J_{3},
\end{array}
$$

or equivalently,

$$
-i k^{-1} \frac{d}{d x}\langle\varphi|=\langle\varphi| N(x) .
$$

In our case, $N$ and $N^{\dagger}$ are related via

$$
N^{\dagger}=R N R^{-1},
$$

where $R=-R^{-1}=\exp \left(i \pi J_{3}\right)=2 i J_{3}$. With use of this relation, we can rewrite the adjoint equation (28) as

$$
i k^{-1} \frac{d}{d x} R|\varphi\rangle=N(x) R|\varphi\rangle .
$$

and find that $R|\varphi\rangle$ and $|\psi\rangle$ obey the same equation. Hence, if $R|\varphi(0)\rangle=|\psi(0)\rangle$, then $R|\varphi(x)\rangle=|\psi(x)\rangle$ for any $x$. 
Now we introduce the density operator as

$$
\rho=|\psi\rangle\langle\varphi|=| \psi\rangle\langle\psi| R,
$$

which follows the evolution equation,

$$
i k^{-1} \frac{d}{d x} \rho=[N, \rho]
$$

The matrix representation of $\rho$ is given as follows:

$$
\rho=\left[\begin{array}{ll}
-\alpha \beta^{*} & |\alpha|^{2} \\
-|\beta|^{2} & \alpha^{*} \beta
\end{array}\right]
$$

It can be parametrized as

$$
\rho=i\left(s_{0} I / 2+s_{1} K_{1}+s_{2} K_{2}+s_{3} J_{3}\right),
$$

where

$$
\begin{aligned}
& s_{0}=\left(\alpha^{*} \beta-\alpha \beta^{*}\right) / i, \\
& s_{1}=\alpha^{*} \beta+\alpha \beta^{*}, \\
& s_{2}=-|\alpha|^{2}+|\beta|^{2}, \\
& s_{3}=|\alpha|^{2}+|\beta|^{2} .
\end{aligned}
$$

Substitution of (27) and (36) into (34) gives the equation of motion for $s=\left(s_{1}, s_{2}, s_{3}\right)$ :

$$
k^{-1} \frac{d s}{d x}=\epsilon \widetilde{\times} s
$$

which resembles to the Bloch equation, except for the use of the $\mathrm{SU}(1,1)$ vector product.

We note that $s_{0}$ is a constant of motion and that the relation

$$
s \sim s=-s_{0}^{2}
$$

holds.

In the case of $s_{0}=0$, the vector $s$ moves on the surface

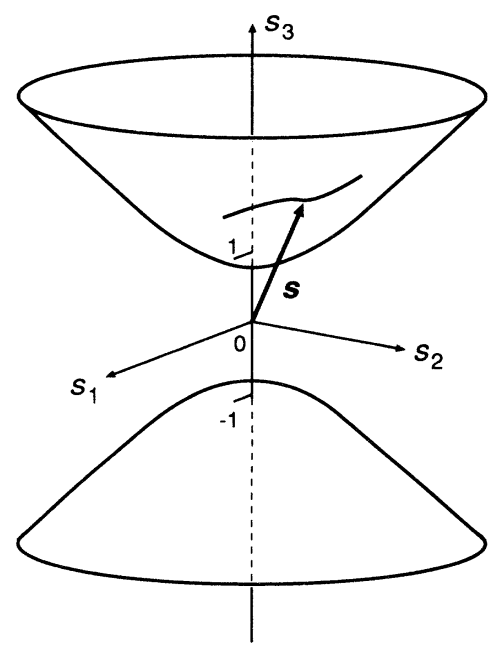

FIG. 5. Bloch vector $s$ (for complex amplitudes) on the hyperboloid: $s_{1}^{2}+s_{2}^{2}-s_{3}^{2}=-1$. of a cone in the $\left(s_{1}, s_{2}, s_{3}\right)$ space, as in Sec. III; $s_{0}=0$ implies real amplitudes because $\alpha$ and $\beta$ share the same phase, which can be eliminated.

In the case of $s_{0} \neq 0, s$ moves on the surface of a twosheet hyperboloid, as shown in Fig. 5. Without loss of generality, we normalize the state vectors as $s_{0}=1$.

\section{GEOMETRICAL PHASE}

In the case of real amplitudes, a density matrix represents two states. On the other hand, in the case of complex amplitudes, a density matrix $\rho$ represents an infinite number of state vectors. For example, the state $\left|\psi^{\prime}\right\rangle=e^{-i \phi K_{s}}|\psi\rangle,\left|\varphi^{\prime}\right\rangle=e^{i \phi K_{s}}|\varphi\rangle$ derived from $|\psi\rangle$ and $|\varphi\rangle$ with $K_{s}=\boldsymbol{s} \cdot \boldsymbol{K}$ yield the same density matrix;

$$
\rho^{\prime}=\left|\psi^{\prime}\right\rangle\left\langle\varphi^{\prime}\left|=e^{-i \phi K_{s}}\right| \psi\right\rangle\langle\varphi| e^{i \phi K_{s}}=\rho,
$$

since $K_{s}$ and $\rho=|\psi\rangle\langle\varphi|=i\left(s_{0} / 2+K_{s}\right)$ commute. In other words, a density matrix or a Bloch vector corresponds to an equivalence class of states: $\left\{e^{-i \phi K_{s}}|\psi\rangle \mid-2 \pi \leq \phi<2 \pi\right\}$, or a ray.

Now we have a projective structure in which we can introduce the Aharonov-Anandan connection [12], thereby we can derive the dynamical phase and the geometrical phase associated with a cycle of evolution.

The equivalence class introduced above can be represented more conveniently by using a decomposition of the group element of $\mathrm{SL}(2, \mathbb{R})$ :

$$
g=e^{-i \mu J_{3}} e^{-i \nu K_{1}} e^{-i \phi J_{3}} .
$$

An arbitrary state $|\psi\rangle$ can be obtained from $\left|\psi_{0}\right\rangle=$ $2^{-1 / 2}(1, i)^{T}$, which belongs to a ray $s=(0,0,1)$ as

$$
|\psi\rangle=g\left|\psi_{0}\right\rangle,\langle\varphi|=\left\langle\varphi_{0}\right| g^{-1},
$$

where $\left|\varphi_{0}\right\rangle=R^{-1}\left|\psi_{0}\right\rangle$. The density matrix for this state is

$$
\rho=|\psi\rangle\left\langle\varphi\left|=e^{-i \mu J_{3}} e^{-i \nu K_{1}}\right| \psi_{0}\right\rangle\left\langle\varphi_{0}\right| e^{i \nu K_{1}} e^{i \mu J_{3}},
$$

which does not contain the parameter $\phi$.

Now we see that all the states in a ray can be derived by applying a group action to the states $\left|\psi_{\phi}\right\rangle=e^{-i \phi J_{3}}\left|\psi_{0}\right\rangle$, whose real and imaginary parts are given as

$$
\left|\psi_{\phi}^{\prime}\right\rangle=\left[\begin{array}{c}
\cos \phi / 2 \\
\sin \phi / 2
\end{array}\right], \quad\left|\psi_{\phi}^{\prime \prime}\right\rangle=\left[\begin{array}{c}
-\sin \phi / 2 \\
\cos \phi / 2
\end{array}\right] .
$$

Thus the element in a ray can be parametrized by $\phi$.

Reflecting the two-valuedness discussed in Sec. IV, the states with $\phi$ and $\phi+2 \pi$ in a ray have opposite signs.

Let us consider a cyclic evolution in which a state $|\psi(0)\rangle$ evolves and returns to a state $|\psi(L)\rangle=$ $\exp (i \Phi)|\psi(0)\rangle$, which belongs to the same ray, i.e., $\rho(0)=$ $\rho(L)$. The phase difference $\Phi$ is composed of a dynamical phase $\phi_{D}$ and a geometrical phase $\phi_{G}$.

The geometrical phase $\phi_{G}$ or the Berry phase for this system is given as $[5,13]$

$$
\phi_{G}=\int_{S} s_{3}^{-1} d s_{1} \wedge d s_{2}
$$


where $S$ is the area enclosed by the path. The integrand is an invariant two form under the group action. It should be noted that this two form is different from that for the surface curvature of the hyperboloid, $\left(2 s_{3}^{2}-1\right)^{-2} d s_{1} \wedge d s_{2}$, or that for the surface area of the hyperboloid, $\left(2-s_{3}^{-3}\right)^{1 / 2} d s_{1} \wedge d s_{2}$. In the $\mathrm{SU}(2)$ case, all of the three quantities have the same form incidentally.

\section{CONCLUSIONS}

In conclusion, we have explored geometrical features of $1 \mathrm{D}$ wave propagation. The underlying group of this problem is the $(2+1)$-dimensional Lorentz group. The geometrical approach would be very helpful for intuitive understanding of various wave phenomena. It is also applicable to other systems that are governed by the Lorentz dynamics.

Finally, we would like to propose an experiment to observe the Berry phase and other geometrical phenomena for the $1 \mathrm{D}$ wave propagation. In Fig. 6, an optical system is presented. We have a medium whose distribution of refractive index $\epsilon(x)$ can be controlled externally. It is possible to write a holographic grating in optical fibers or in some nonlinear media. The light propagated through the medium is reflected back with perfect mirror $M$. Thus we can prepare a state corresponding to $s(0)=(0,0,1)$ on the mirror side $(x=0)$. By adjusting the mirror position, we can "tune" the phase $\phi_{0}$ of the initial state as

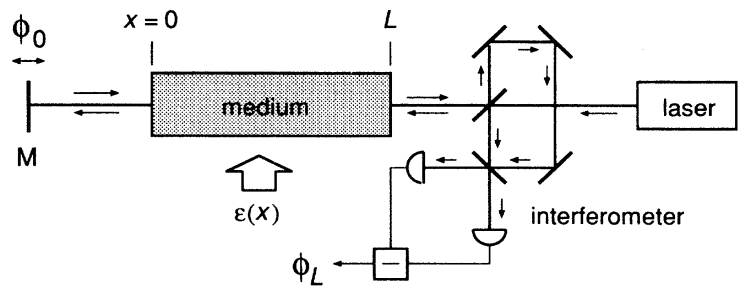

FIG. 6. Proposed experimental setup for measuring the Berry phase for a $1 \mathrm{D}$ wave system.

$|\psi(0)\rangle=\left(\cos \phi_{0} / 2, \sin \phi_{0} / 2\right)^{T}$ and hence we can map out the complex $|\psi\rangle$ via Eq. (44). If we set up $\epsilon(x)$ so that the evolution be cyclic, then the state on the other side $(x=L)$ should be $|\psi(L)\rangle=\left(\cos \phi_{L} / 2, \sin \phi_{L} / 2\right)^{T}$. For a cyclic evolution, $\Phi=\phi_{L}-\phi_{0}$ is independent of $\phi_{0}$. With the Mach-Zehnder interferometer we can measure the difference $\Phi=\phi_{G}+\phi_{D}$.

\section{ACKNOWLEDGMENTS}

The author is grateful to Professor H. Ogura for valuable discussions. This work is partly supported by the Ministry of Education, Science, and Culture in Japan, under a Grant-in-Aid for Scientific Research.
[1] M. V. Berry, Proc. R. Soc. London, Ser. A 392, 45 (1984).

[2] R. Y. Chiao and T. F. Jordan, Phys. Lett. A 132, 77 (1988).

[3] B. Yurke, S. McCall, and J. R. Klauder, Phys. Rev. A 33, 4033 (1986).

[4] D. Han, E. E. Hardekopf, and Y. S. Kim, Phys. Rev. A 39, 1269 (1989).

[5] M. Kitano and T. Yabuzaki, Phys. Lett. A 142, 321 (1989).

[6] G. Dattioli, A. Dipace, and A. Torre, Phys. Rev. A 33 , 4387 (1986)

[7] H. Rauch, A. Zeilinger, G. Badurek, A. Wilfing, W. Bauspiess, and U. Bonse, Phys. Lett. 54A, 425 (1975); S. A.
Werner, R. Colella, A. W. Overhauser, and C. F. Eagen, Phys. Rev. Lett. 35, 1053 (1975).

[8] H. C. Longuet-Higgins, Proc. R. Soc. London, Ser. A 344, 147 (1975).

[9] M. V. Berry and M. Wilkinson, Proc. R. Soc. London, Ser. A 392, 15 (1984).

[10] J. M. Leinaas and J. Myrheim, Nuovo Cimento B 37, 1 (1977).

[11] J. C. Garrison and E. M. Wright, Phys. Lett. 128, 177 (1988).

[12] Y. Aharonov and J. Anandan, Phys. Rev. Lett. 58, 1593 (1987).

[13] T. F. Jordan, J. Math. Phys. 29, 2042 (1988). 